\title{
Analysis of Multiresonance and Chaotic Behavior of the Polarization in Materials Modeled by a Duffing Equation with Multifrequency Excitations
}

\author{
C. Ainamon ${ }^{1}$, C. H. Miwadinou ${ }^{2}$, A.V. Monwanou ${ }^{2} \&$ J. B. Chabi Orou ${ }^{2,3}$ \\ ${ }^{1}$ Ecole Doctorale Sciences des Matériaux, Université d'Abomey - Calavi, Bénin \\ ${ }^{2}$ Institut de Mathématiques et de Sciences Physiques, Université d'Abomey - Calavi, Bénin \\ ${ }^{3}$ Département de Physique, Université d'Abomey - Calavi, Bénin \\ Correspondence: A. V. MONWANOU, Institut de Mathématiques et de Sciences Physiques, 01 BP 613 \\ Porto-Novo, Bénin. E-mail: movins2008@yahoo.fr; vincent.monwanou@imsp-uac.org
}

Received: October 10, 2014 Accepted: October 27, 2014 Online Published: November 14, 2014

doi:10.5539/apr.v6n6p74 URL: http://dx.doi.org/10.5539/apr.v6n6p74

\begin{abstract}
This paper considers nonlinear dynamics of polarization oscillations when some materials are subjected to the action of an electromagnetic wave modeled by multifrequency forced Duffing equation. Multiresonance and chaotic behavior are analyzed. For the resonance analysis, the method of multiple scales is used. The phenomena of amplitude jump and hysteresis for polarization were observed and analyzed. Finally, the study of chaotic behavior for polarization was made by numerical simulation using the Runge-Kutta fourth order algorithm.
\end{abstract}

Keywords: multiresonance, jump phenomena, hysteresis, polarization, bifurcation, chaotic, Duffing oscillator

\section{Introduction}

We consider the Duffing oscillator equation which models a material submitted to multifrequency excitation of an electromagnetic wave (Aïnamon, Miwadinou \& Chabi Orou, 2014)

$$
\ddot{P}+2 \varepsilon \mu \dot{P}+P+\varepsilon P^{3}=F_{01} \cos \omega_{1} t+F_{02} \cos \omega_{2} t .
$$

The interaction of light with a non-linear optical material changes the properties of the material and thereby alters the frequency, phase or polarization of the light passed through. This is the cause of significant non-linear optical phenomena including the optical Kerr effect and frequency doubling (Boyd, R., 2007). Resonance plays an important role in non-linear physics problems specifically in the study of non-linear behavior of a material. The response of a nonlinear system to a weak periodic signal can be enhanced by means of an appropriate noise, a high frequency periodic force, or a chaotic signal (Gouttenoireet al., 2010; Nayfeh, 1981; Steven \& Strogatz, 1994; Thomas, Nezamabadi, Lazarus,, \& Deü, 2013; Wiggins, 1990; Yang \& Liu, 2010; Yang, Sanjuán, Wang, \& Zhu, 2013; Yao \& Zhan, 2010; Yao, Liu, \& Zhan, 2011). The enhancements of the response of a system due to the applied weak noise, a high frequency force, or a chaotic signal are termed as stochastic resonance, vibrational resonance, and chaotic resonance, respectively. Among them, much attention has been given to stochastic resonance and a lot of progress has been made. However, the analysis of multiresonance has also received considerable interest in recent years due to its importance in a wide variety of contexts in physics, engineering, and biology. On the other hand, so far, it has been seen that the presence of chaos in many systems has been extensively demonstrated and is very common. The purpose of this paper is to take into account the multiresonance in the modeling of Equation (1) and then to investigate using analytical methods, harmonic and resonant states which can be displayed by the model in such conditions. We also aim to perform the possible bifurcation mechanisms of the model using numerical tools. The phase portraits and basin of attraction corresponding to the bifurcation diagram and its corresponding Lyapunov have been plotted.

The paper is organized as follows. Sec. 2, addresses the multiresonant states of the model through the multiple time scales method. In Sec. 3, we point out bifurcation and chaotic sequences of the model. We conclude in Sec. 4 . 


\section{Multiresonant States}

Generally, many types of oscillations can be found in a forced system additionally, to the harmonic oscillatory states. Such oscillation occurs when the external frequency is too close or far from the internal frequency, according also to the external excitation strength. Since these oscillations rise up at different time scale, the best tool to be used for their investigation is the multiple time method (Aïnamon, Miwadinou, \& Chabi Orou, 2014; Gammaitoni, Hänggi, Jung, \& Marchesoni, 1998; Gang, Ditzinger, Ning, \& Haken, 1993; Nayfeh, 1981). In such a situation, an approximate solution is generally sought as follow

$$
P(t, \varepsilon)=P_{o}\left(T_{o}, T_{1}\right)+\varepsilon P_{1}\left(T_{o}, T_{1}\right)+\cdots,
$$

where the fast time scale to end the slow time scale are associated respectively to the unperturbed system and to the amplitude and phase induced by the global first order perturbation. The first and second time derivatives can now be rewritten as follow:

$$
\begin{gathered}
\frac{d}{d t}=D_{o}+\varepsilon D_{1}+\ldots, \frac{d^{2}}{d t^{2}}=D_{o}^{2}+2 \varepsilon D_{o} D_{1}+\varepsilon^{2} D_{1}^{2}+\cdots, \\
\text { with } D_{n}=\frac{\partial}{\partial T^{n}}, T_{n}=\varepsilon^{n} t ; n=0,1,2, \ldots .
\end{gathered}
$$

\subsection{Primary Resonant State}

In this state, we set $F_{01}=\varepsilon F_{1}, F_{02}=\varepsilon F_{2}$. The external frequencies are given by $\omega_{1} \simeq \omega_{2} \simeq 1+\varepsilon \sigma$.

Inserting these relations in Equation (1), we obtained

$$
D_{o}^{2} P_{o}+P_{o}=0
$$

and

$$
D_{o}^{2} P_{1}+\omega_{0}^{2} P_{1}=-2 D_{o} D_{1} P_{0}-2 \mu D_{o} P_{0}-\lambda P_{0}^{3}+F_{1} \cos \omega_{1} T_{0}+F_{2} \cos \omega_{2} T_{0}
$$

The general solution of Equation (4) is:

$$
P_{0}=A e^{i \omega_{0} T_{o}}+C C
$$

with $\mathrm{A}=\frac{1}{2} a\left(T_{1}\right) e^{i \theta}$. Inserting Equation (6) into Equation (5) we get

$$
D_{o}^{2} P_{1}+\omega_{0}^{2} P_{1}=\left[-2 i \omega_{0} A^{\prime}-2 i \mu \omega_{0} A-3 \lambda A^{2} \bar{A}+\frac{1}{2} F_{1} e^{i \sigma T_{1}}+\frac{1}{2} F_{2} e^{i \sigma t_{1}}\right] e^{i \omega_{0} T_{o}}+N S T+C C .
$$

By vanishing the secular terms, we obtain:

$$
-2 i \quad A^{\prime}-2 i \mu A-3 \lambda A^{2} \bar{A}+\frac{F}{2} e^{i \sigma T_{1}}=0
$$

where $F=F_{1}+F_{2}, \omega_{0}=1$.

Injecting A in Equation (8) and separating real and imaginary terms, we obtain the following system:

$$
\begin{aligned}
& a^{\prime}=-\mu \mathrm{a}+\frac{F}{2} \sin \emptyset, \\
& a \theta^{\prime}=\frac{3}{8} \lambda a^{3}-\frac{F}{2} \cos \emptyset,
\end{aligned}
$$

where $\varnothing=\sigma T_{1}-\theta$.

Finally, we obtain the coupled flow for the amplitude and phase

$$
\begin{aligned}
& a^{\prime}=-\mu \mathrm{a}+\frac{F}{2} \sin \emptyset, \\
& a \emptyset^{\prime}=a \sigma-\frac{3}{8} \lambda a^{3}+\frac{F}{2} \cos \emptyset .
\end{aligned}
$$

The steady state conditions ( $\left.\quad a^{\prime}=\emptyset^{\prime}=0\right)$ imply:

$$
\mu \mathrm{a}=\frac{F}{2} \sin \emptyset,
$$




$$
a \sigma-\frac{3}{8} \lambda a^{3}=-\frac{F}{2} \cos \emptyset
$$

Eliminating $\varnothing$ in Equation (12) and (13), we obtain the following nonlinear algebraic equation

$$
\mu^{2} a_{0}^{2}+\left(a_{0} \sigma-\frac{3}{8} \lambda a_{0}^{3}\right)^{2}=\frac{F^{2}}{4},
$$

where $a_{0}$ and $\emptyset_{0}$ are respectively the values of $a$ and $\varnothing$ on the steady-state.

Figure 1 shows a representative curve, called a frequency-response curve, for the variation of $a$ with $\sigma$. The bending of the frequency-response curve is responsible for a jump phenomenon. To explain this, we imagine that an experiment is performed in which the amplitude of the excitation is held fixed, the frequency of the excitation (i.e. $\sigma$ ) is very slowly varied up and down through the linear natural frequency, and the amplitude of the harmonic response is observed. The experiment is started at a frequency corresponding to point $\mathrm{A}$ on the curve in Figure 1. As frequency is reduced, $\sigma$ decreases and $a$ slowly increases through point $\mathrm{B}$ until point $\mathrm{C}$ is reached. As $\sigma$ is decreased further, a jump from point $\mathrm{C}$ to point $\mathrm{D}$ takes place with an accompanying increase of $a$, after which $a$ decreases slowly with decreasing $\sigma$. If the experiment is started at point $\mathrm{E}$ and $\sigma$ is increased, $a$ increases slowly through point $\mathrm{D}$ until point $\mathrm{F}$ is reached. As $\sigma$ increased further, $a$ jump from point $\mathrm{F}$ to $\mathrm{B}$ takes place with an accompanying decrease of $a$, after which $a$ decreases slowly with increasing $\sigma$. The maximum amplitude corresponding to $\mathrm{F}$ is reached only when approached a lower frequency. The portion of the response curve between points $\mathrm{C}$ and $\mathrm{F}$ is unstable, and hence, cannot be produced experimentally.

If the experiment is performed with the frequency of the excitation $\omega_{1}=\omega_{2}$ held fixed while the amplitude of excitation is varied slowly, a similar jump phenomenon can be observed. Suppose that the experiment is started at point $A$ in Figure 2. As $F_{1}+F_{2}$ is increased, a slowly increases through point $B$ to point $C$. As $F_{1}+F_{2}$ is increased further, a jump takes place from point $\mathrm{C}$ to point $\mathrm{D}$, with accompanying increase of $a$, after which $a$ increases slowly with $F_{1}+F_{2}$. If the process is reserved, $a$ decreases slowly as $F_{1}+F_{2}$ decreases from point $E$ to point $F$. As $F_{1}+F_{2}$ is decreased further, a jump from point $F$ to point $B$ takes place, with an accompanying decrease in $a$, after which $a$ decreases slowly with decreasing $\mathrm{F}_{1}+\mathrm{F}_{2}$. Figure1 (b) and Figure 2 (b) show the effect on jump and hysteresis phenomena of $\mathrm{F}=\mathrm{F}_{1}+\mathrm{F}_{2}$ and $\mu$ respectively. Through these figures we notice that the excitation amplitude or damping parameter can be used to control the polarization oscillation in material.
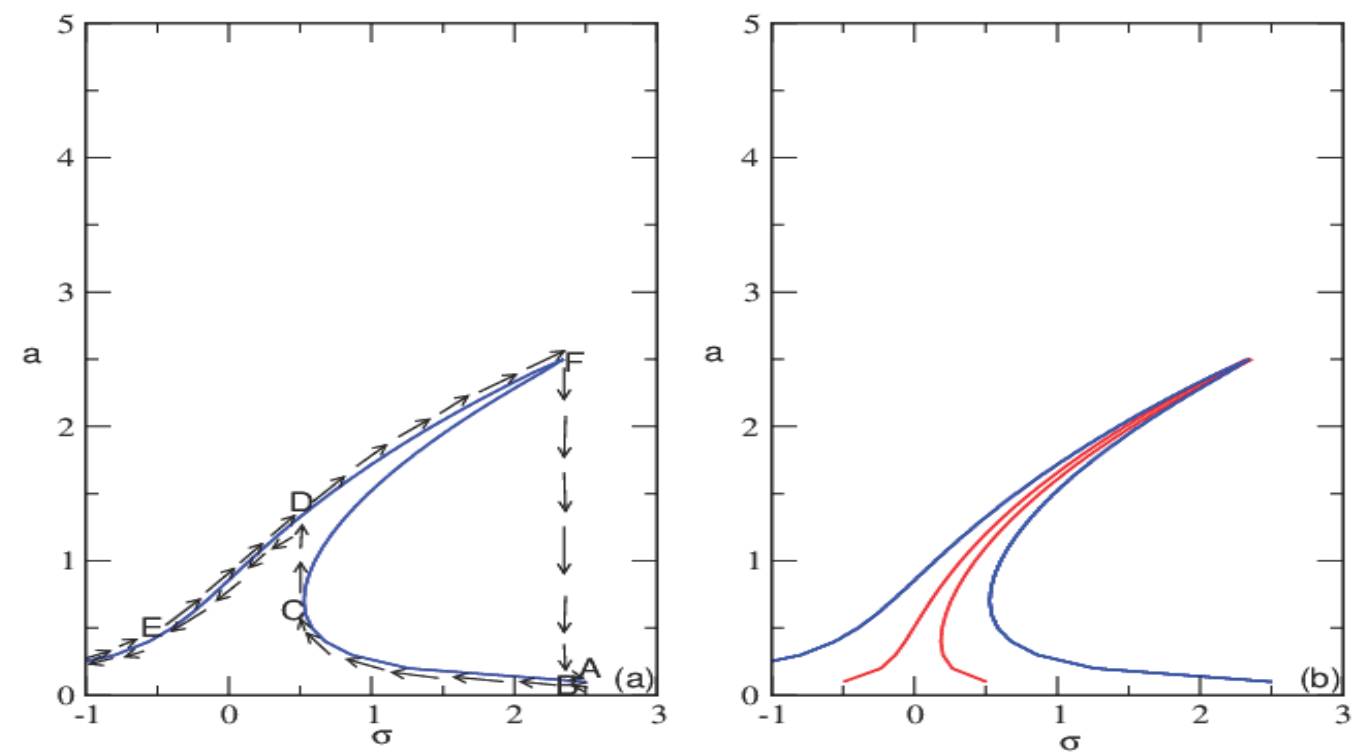

Figure 1. Jump phenomena for primary resonance of the polarization in the system for $\mu=0.004, \lambda=$ 1; with (a): $F_{1}=F_{2}=F=0.14$ and (b): The effects of parameter $\mathrm{F}$ 

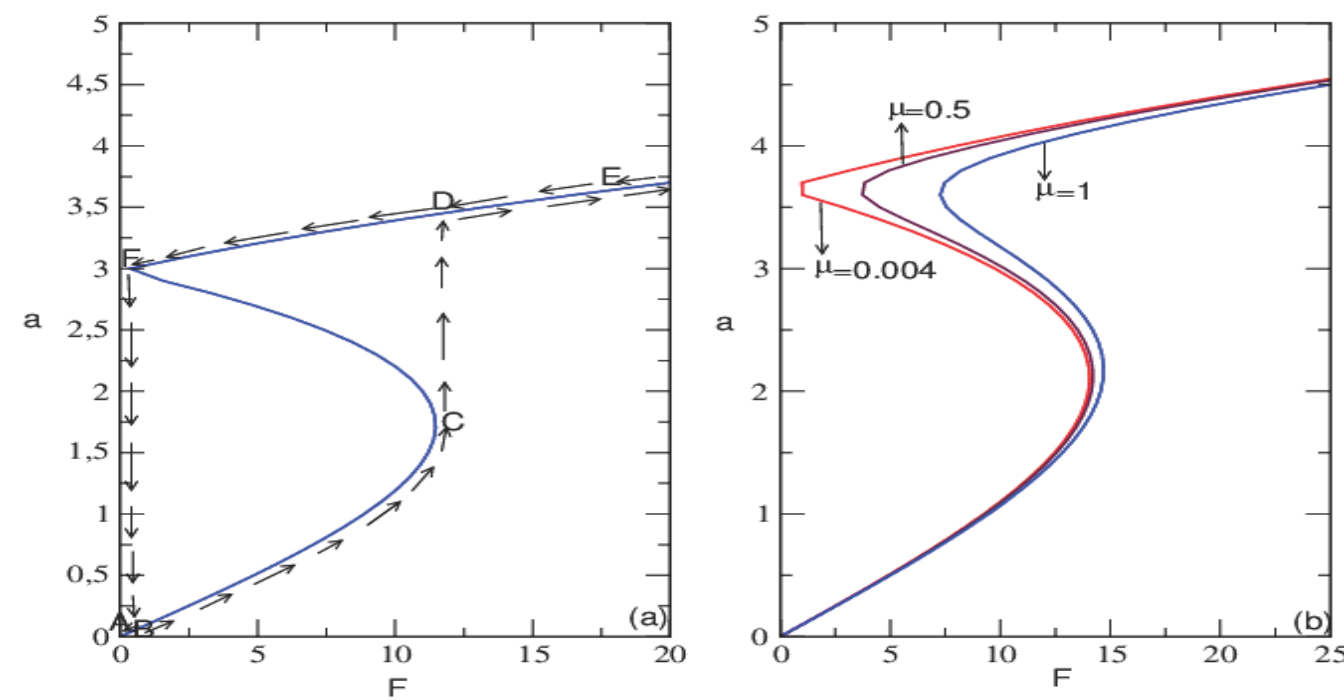

Figure 2. (a):Jump phenomena for primary resonance of the polarization in the system for the parameters of Figure 1 and (b): The effects of parameter $\mu$

\subsection{Other Resonant States}

When the amplitude of the sinusoidal external force is larger than that of the precedent case, other type of oscillations can be displayed by the model, namely the superharmonic, the subharmonic oscillatory states (Aïnamon, Miwadinou, \& Chabi Orou, 2014) and other secondary resonances states. It is now assumed that $F=\varepsilon^{0} F$ and therefore, one obtains the following equations at different order of $\varepsilon$.

Order $\varepsilon^{0}$

$$
D_{o}^{2} P_{0}+P_{0}=F_{01} \cos \omega_{1} t_{o}+F_{02} \cos \omega_{2} t_{o}
$$

Oder $\varepsilon^{1}$

$$
D_{o}^{2} P_{1}+P_{1}=-2 D_{o} D_{1} P_{o}-2 \mu D_{o} P_{o}-\lambda P_{o}^{3}
$$

From Equation (15), we have

$$
\begin{aligned}
& P_{0}=+A e^{i T_{o}}+\bar{A} e^{-i T_{o}}+\Lambda_{1} e^{i \omega_{1} T_{o}}-3 \lambda \bar{A} \Lambda_{1}^{2} e^{i \omega_{1} T_{o}}+\Lambda_{2} e^{i \omega_{2} T_{o}} \Lambda_{2} e^{-i \omega_{2} T_{o}}, \\
& \text { with } \wedge_{1}=\frac{F_{01}}{2\left(1-\omega_{1}^{2}\right)}, \Lambda_{2}=\frac{F_{02}}{2\left(1-\omega_{2}^{2}\right)} \text {, and } \mathrm{A}=\frac{1}{2} a\left(T_{1}\right) e^{i \theta} .
\end{aligned}
$$

Substituting the general solution $P_{0}$ into Equation(16) we get

$$
\begin{aligned}
D_{o}^{2} P_{1}+P_{1}= & {\left[-2 i\left(A^{\prime}+\mu A\right)-3 \lambda A^{2} \bar{A}-6 \lambda \wedge_{2}^{2} A-6 \lambda A \wedge_{1}^{2}\right] e^{i T_{0}+} } \\
& +\left[-2 i \mu \wedge_{1}-6 \lambda A \bar{A} \wedge_{1}-6 \lambda \wedge_{1} \wedge_{2}^{2}-3 \lambda \wedge_{1}^{3}\right] e^{i \omega_{1} T_{0}+} \\
& +\left[-2 i \mu \wedge_{2}-6 \lambda \wedge_{2} A \bar{A}-3 \lambda \wedge_{2}^{3}-6 \lambda \wedge_{2} \wedge_{1}^{2}\right] e^{i \omega_{2} T_{0}+} \\
& +-3 \lambda \wedge_{2} \bar{A}^{2} e^{i\left(-2+\omega_{2}\right) T_{o}}-3 \lambda \bar{A} \wedge_{2}^{2} e^{i\left(-1+2 \omega_{2}\right) T_{o}+} \\
& +-3 \lambda \wedge_{1} \bar{A}^{2} e^{i\left(-2+\omega_{1}\right) T_{o}}-3 \lambda A^{3} e^{3 i T_{o}}-3 \lambda \wedge_{1}^{3} e^{3 i \omega_{1} T_{o}+} \\
& -3 \lambda \wedge_{2}^{3} e^{3 i \omega_{2} T_{o}}-6 \lambda \wedge_{1} \wedge_{2} e^{i\left(1-\omega_{1}+\omega_{2}\right) T_{o}+} \\
& -6 \lambda A \wedge_{1} \wedge_{2} e^{i\left(1+\omega_{1}-\omega_{2}\right) T_{o}}-6 \lambda \bar{A} \wedge_{1} \wedge_{2} e^{i\left(-1+\omega_{1}+\omega_{2}\right) T_{o}+} \\
& -3 \lambda \wedge_{2} A^{2} e^{i\left(2-\omega_{2}\right) T_{o}}-3 \lambda \wedge_{1} A^{2} e^{i\left(2-\omega_{1}\right) T_{o}+} \\
& +-3 \lambda \wedge_{1} \wedge_{2} e^{i\left(2 \omega_{2}-\omega_{1}\right) T_{o}}-3 \lambda \bar{A} \wedge_{1}^{2} e^{i\left(-1+2 \omega_{1}\right) T_{o}}+ \\
& -3 \lambda \wedge_{2} \wedge_{1}^{2} e^{i\left(-\omega_{2}+2 \omega_{1}\right) T_{o}}-3 \lambda \bar{A} \wedge_{2}^{2} e^{i\left(-1+2 \omega_{2}\right) T_{o}}+C C+N S T
\end{aligned}
$$

where CC denotes the complex conjugate and NST the non-secular terms.

The particular solutions of $P_{1}$ contain secular and small divisions terms. For the uniform expansion, the secular and small division terms must be eliminated by proper choices depending on the type of resonances. The cases of superharmonic $\left(3 \omega_{1}=3 \omega_{2}=1+\sigma \varepsilon\right)$ and suharmonic $\left(\omega_{1}=\omega_{2}=3+\sigma \varepsilon\right)$ are treated in a previous work 
(Aïnamon, Miwadinou, \& Chabi Orou, 2014). In the present work, we treat the three cases for secondary resonances: $\omega_{1}+\omega_{2}=2,2 \omega_{2}-\omega_{1}=1$ and $2 \omega_{1}-\omega_{2}=1$.

2.2.1 Case $\omega_{1}+\omega_{2}=2$

In this case, we assume that $\omega_{1}+\omega_{2}=2$ and no other resonances exist at first order. To describe quantitatively the resonance state when $\omega_{1}+\omega_{2}=2$, we introduce the detuning parameter $\sigma$ defined by $\omega_{1}+\omega_{2}=2+\sigma \varepsilon$. In this case, Equation (19) can be rewritten as follow

$$
\left.D_{o}^{2} P_{1}+P_{1}=\left[\begin{array}{ll}
-2 i\left(A^{\prime}+\mu\right. & A
\end{array}\right)-3 \lambda A^{2} \bar{A}-6 \lambda A \Lambda_{2}^{2}+-6 \lambda \Lambda_{1}^{2} A\right] e^{i T_{0}}-6 \lambda \bar{A} \Lambda_{1} \Lambda_{2} e^{i\left(-1+\omega_{1}+\omega_{2}\right) T_{0}}+C C+N S T .
$$

With this condition, we rewrite Equation (20) as

$$
D_{o}^{2} P_{1}+P_{1}=\left[-2 i\left(A^{\prime}+\mu A\right)-3 \lambda A^{2} \bar{A}-6 \lambda A \Lambda_{2}^{2}+-6 \lambda \wedge_{1}^{2} A\right] e^{i T_{0}}-6 \lambda \bar{A} \Lambda_{1} \wedge_{2} e^{i \sigma T_{1}} e^{i T_{0}} .
$$

Eliminating the secular terms from Equation (21), we get

$$
-2 i\left(A^{\prime}+\mu A\right)-3 \lambda A^{2} \bar{A}-6 \lambda A \wedge_{2}^{2}-6 \lambda \wedge_{1}^{2} A+-6 \lambda \bar{A} \wedge_{1} \wedge_{2} e^{i \sigma T_{1}}=0 .
$$

Equating real and imaginary parts to 0 we obtain:

$$
\begin{gathered}
-a^{\prime}-\mu a-3 \lambda \wedge_{1} \wedge_{2} a \cos \emptyset=0, \\
a \theta^{\prime}-\frac{3}{8} \lambda a^{3}-3 \lambda\left(\wedge_{1}^{2}+\Lambda_{2}^{2}\right) a-3 \lambda \wedge_{1} \wedge_{2} a \sin \emptyset=0,
\end{gathered}
$$

with $\emptyset=\sigma T_{1}-2 \theta$.

The system (23) becomes

$$
\begin{gathered}
a^{\prime}=-\mu a-3 \lambda \wedge_{1} \wedge_{2} a \cos \emptyset \\
a \theta^{\prime}=a \sigma-\frac{6}{8} \lambda a^{3}-6 \lambda\left(\wedge_{1}^{2}+\Lambda_{2}^{2}\right) a-6 \lambda \wedge_{1} \wedge_{2} \sin \emptyset
\end{gathered}
$$

and leads to

$$
\begin{gathered}
-3 \mu a_{s}-6 \lambda \wedge_{1} \wedge_{2} a_{s} \cos \emptyset=0, \\
a_{s} \sigma-\frac{6}{8} \lambda a_{s}^{3}-6 \lambda\left(\wedge_{1}^{2}+\Lambda_{2}^{2}\right) a_{s}=-6 \lambda \wedge_{1} \wedge_{2} a_{s} \sin \emptyset,
\end{gathered}
$$

by setting $a^{\prime}=0$ and $\emptyset^{\prime}=0$

The amplitude of this resonance state is governed by the following nonlinear algebraic equation

$$
4 \mu^{2} a_{s}^{2}+\left(a_{s} \sigma-\frac{6}{8} \lambda a_{s}^{3}-6 \lambda\left(\Lambda_{1}^{2}+\Lambda_{2}^{2}\right) a_{s}\right)^{2}=36 \lambda^{2} \Lambda_{1}^{2} \wedge_{2}^{2} a_{s}^{2} .
$$

Equation (26) can be rewritten as follow

$$
\begin{aligned}
& 4 \mu^{2}+\left(\sigma-\frac{3}{4} \lambda a_{s}^{2}-6 \lambda\left(\Lambda_{1}^{2}+\Lambda_{2}^{2}\right) a_{s}\right)^{2}=36 \lambda^{2} \Lambda_{1}^{2} \Lambda_{2}^{2} . \\
& \emptyset_{s}=\frac{1}{2} \sigma \varepsilon t-\left\{\frac{1}{2} \tan ^{-1}-\frac{1}{2 \mu}\left[\sigma-\frac{3}{4} \lambda a_{s}^{2}-6 \lambda\left(\Lambda_{1}^{2}+\Lambda_{2}^{2}\right)\right\} .\right.
\end{aligned}
$$

We conclude that at order 1 , the polarization is defined by

$$
P_{0}=a_{s} \exp i \theta_{s} \cos (t)+\frac{F_{1}}{1-\omega_{1}^{2}} \cos \omega_{1} t+\frac{F_{2}}{1-\omega_{2}^{2}} \cos \omega_{2} t,
$$

where $a_{s}$ and $\theta_{s}$ are defined respectively by Equation (27) and Equation (28).

For this order, the susceptibility is defined through the following relation

$$
\begin{gathered}
P_{0} \simeq E_{0} \chi\left(\omega_{i}\right) E\left(\omega_{i}\right), i=1,2 \\
\chi\left(\omega_{i}\right)=\frac{P_{0}}{E_{0} E\left(\omega_{i}\right)},
\end{gathered}
$$

where $P_{0}$ is defined by Equation (29). 
In Figure 3 we plotted the amplitude-response curve. It is found that with this resonant state, the polarization of material exhibits the highest hysteresis phenomenon. This can induce dangerous or undesirable behaviors in material because the polarization and the susceptibility of a material might be subjected to a singularity which could lead to chaotic or catastrophic behaviors.

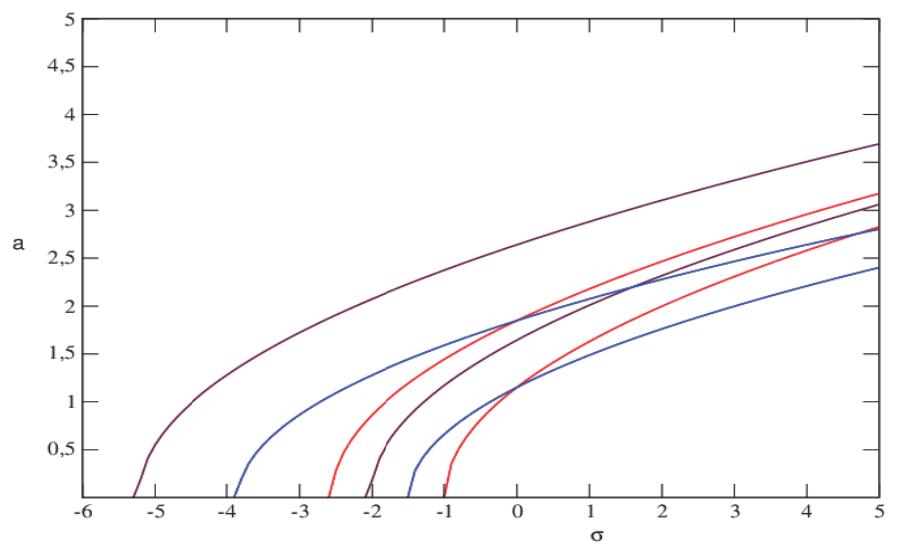

Figure 3. Effects of amplitude of external forced on the resonance case $\omega_{1}+\omega_{2}=2$

\subsubsection{Cases $2 \omega_{1}-\omega_{2}=1+\varepsilon \sigma$ or $2 \omega_{2}-\omega_{1}=1+\varepsilon \sigma$}

Here, we assume that $2 \omega_{1}-\omega_{2}=1+\varepsilon \sigma$. From this relation, Equation (19) can be rewritten as follow:

$$
D_{o}^{2} P_{1}+P_{1}=\left(-2 i\left(A^{\prime}+\mu A\right)-3 \lambda A^{2} \bar{A}-6 \lambda \wedge_{2}^{2} A+-6 \lambda \wedge_{1}^{2} A\right) e^{i T_{0}}-3 \lambda \Lambda_{1} \wedge_{2}^{2} e^{i \sigma \varepsilon T_{0}} \times e^{i T_{0}}+c c+N S T .
$$

Eliminating the secular terms from Equation (32) and equating real and imaginary parts to 0, we obtain

$$
\begin{gathered}
a^{\prime}=-\mu a-3 \lambda \wedge_{1} \wedge_{2}^{2} \cos \emptyset \\
a \theta^{\prime}=\frac{3}{8} \lambda a^{3}+3 \lambda\left(\wedge_{1}^{2}+\wedge_{2}^{2}\right) a+3 \lambda \wedge_{1} \wedge_{2}^{2} \sin \emptyset
\end{gathered}
$$

with $\emptyset=\sigma T_{1}-\theta$.

Steady-state solutions are sought, the amplitude of this resonance state is governed by the following nonlinear algebraic equation

$$
\mu^{2} a_{s}^{2}+\left[\sigma-\frac{3}{8} \lambda a_{s}^{2}-3 \lambda\left(\wedge_{1}^{2}+\wedge_{2}^{2}\right)\right]^{2}-9 \lambda^{2} \wedge_{1}^{2} \wedge_{2}^{4}=0
$$

In Figure 4 we have plotted the amplitude-response curve. We found the same behaviors as in the previous case.

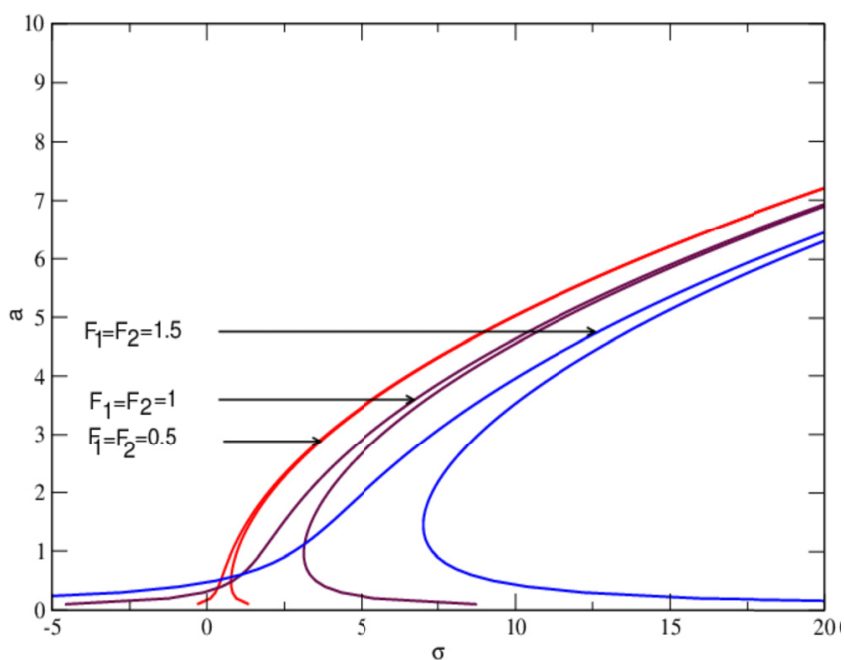

Figure 4. Jump phenomena for resonance states $2 w_{2}-w_{1} \simeq 1$ or $2 w_{1}-w_{2} \simeq 1$ of the polarization in the system for the parameters of Figure1; (a): $w_{2}=1.5 ; w_{1}=2$ and (b): The effects of parameter $\mathrm{F}$ 


\section{Bifurcation and Chaotic Behaviors}

To illustrate the dynamical behavior of the system it is necessary to simulate the original equations. Here, to identify the chaotic motion for resonant states, we have used the Runge-Kutta method of the fourth order to solve Equation (1). By varying the parameters of system, we draw the resulting bifurcation diagram and the variation of the corresponding largest Lyapunov exponent versus amplitude $\mathrm{F}=\mathrm{F}_{1}=\mathrm{F}_{2}$. The Lyapunov exponent is defined as (Enjieu, Chabi Orou \& P. Woafo, 2008a, Enjieu, Nana, Chabi Orou \& Talla, 2008b):

$$
\text { Lya }=\lim _{t \rightarrow \infty} \frac{\ln \sqrt{d P^{2}+d v_{P}^{2}}}{t}
$$

where $d P$ and $d v_{P}$ are the variations of $P$ and $\dot{P}$ respectively.

In our numerical code we started calculations from the same initial conditions $\left(P_{0}=1, \dot{P}_{0}=1\right)$. It is found that the model can switch from quasi-periodic oscillations to chaotic states or from chaotic to quasi-periodic oscillations when the amplitude of external excitation is varied as shown in the bifurcation diagram and its corresponding Lyapunov exponent diagram (see Figures 5 and 6). In order to illustrate such situations, we have represented the phase portraits using the parameters of the corresponding bifurcation diagrams. With appropriate choice of the amplitude of the external excitation F, the phase portraits (chaotic and quasi-periodic motion) are observed in Figures 7 and 8 respectively for primary resonant state (Figure 5) and other resonant state $2 \omega_{1}-\omega_{2}=1$ (Figure 6). We noticed that the polarization behavior is very chaotic on a large domain in the last case (see Figures 6 and 8). Among these domains, we have the following $F \in[2.1,14.5]$. It should be emphasized from Figure 6 that there are some domains where the Lyapunov exponent does not match very well the regime of oscillations expected from the bifurcation diagram. Far from being an error which has occurred from the numerical simulation process, such a behavior corresponds to what is called the intermittency phenomenon. Since the model is highly sensitive to the initial conditions, it can leave quasi-periodic state for a chaotic state without changing the physical parameters. Therefore, its basin of attraction has been plotted (see Figure 9) in order to situate some regions of initial conditions for which chaotic oscillations are observed. In that Figure, the blue zone stands for the area where the choice of the initial conditions lead to a chaotic motion while the white area is the domain of periodic or quasi-periodic oscillations. The same analysis are noticed through Figures 10,11 and 12 and 13 which shown the effects of parameters $\mu, \varepsilon$ and external forcing amplitude.

Figures 13 and 14 are obtained when $\omega_{1}+\omega_{2} \simeq 2$ and $2 \omega_{1}-\omega_{2} \simeq 1$ respectively with $\omega_{1}=\omega_{2}=1$. Comparing these Figures with Figures $12 \mathrm{~b}$ ) and $11 \mathrm{~d}$ ) respectively, we noticed that the polarization of a material subjected to the action of a multifrequency electromagnetic wave is more chaotic when both frequencies are of the same magnitude than when they are different. This remark is confirmed by phase portraits plots. Polarization depends on the susceptibility of the material that allows the deduction of electrical, physical, mechanical ... properties of the material. The highly chaotic state of polarization when external frequencies are different results in a non-symmetrical susceptibility tensor leading to symmetry breaking (Figures 3 and 4). Thus the phase modulating, self-phase modulation, period doubling and Kerr-optic phenomenon properties will not be obtained. These various properties are easily derived in the case where the external frequencies are the same. From these chaotic basins, we notice that with these resonant states, the polarization of material exhibits dangerous or undesirable behaviors. 

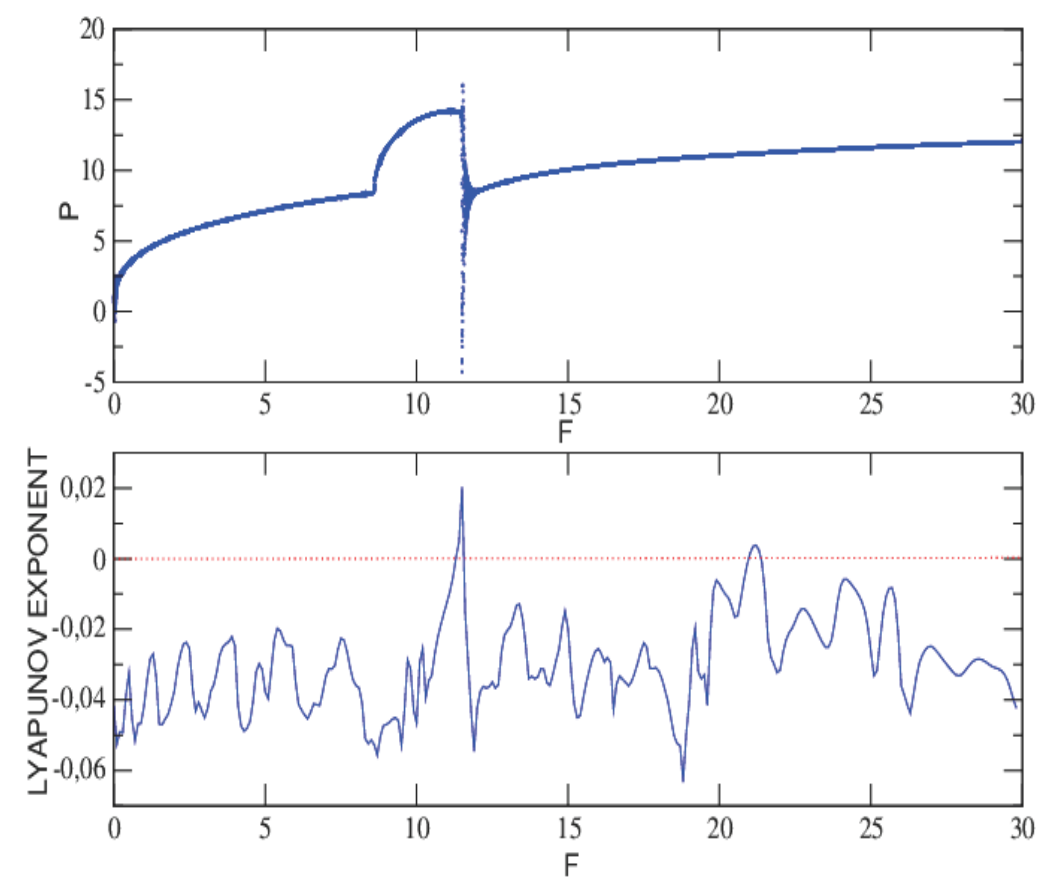

Figure 5. Bifurcation diagram (upper frame) and Lyapunov exponent (lower frame) versus the amplitude $F$ with the parameters $\mu=0.05, \lambda=1, \varepsilon=0.02$ and $\omega_{1} \simeq \omega_{2} \simeq 1$
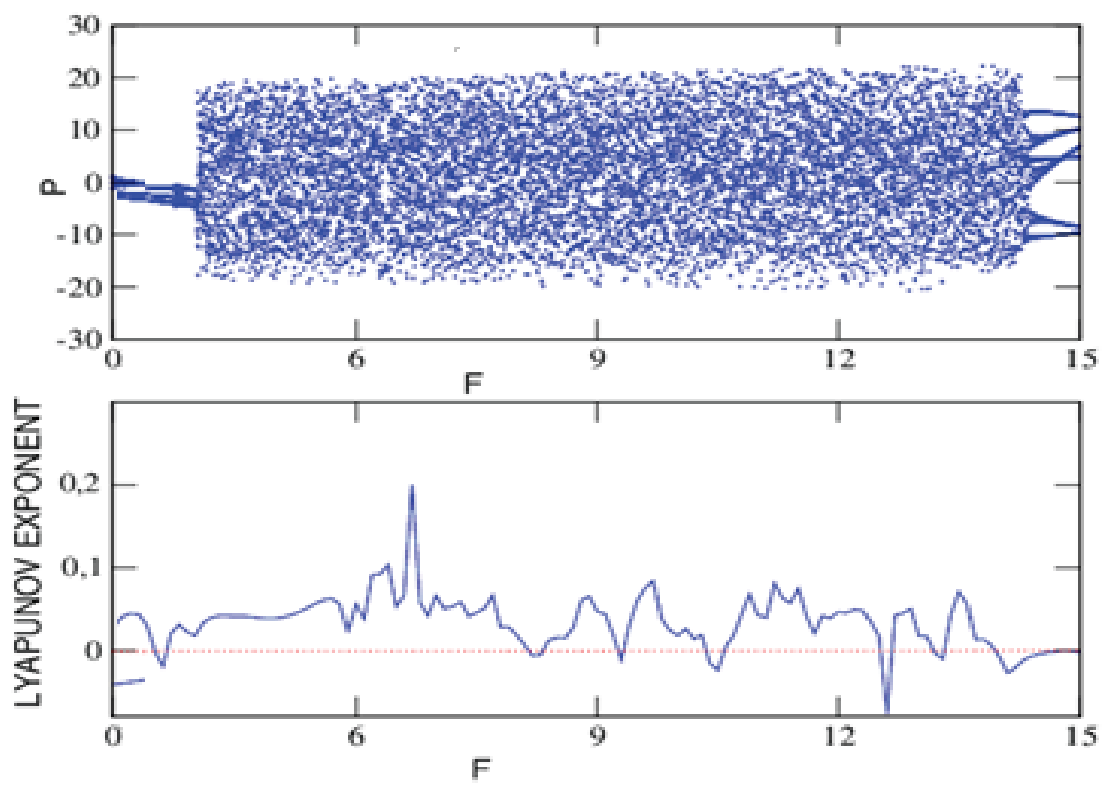

Figure 6. Bifurcation diagram (upper frame) and Lyapunov exponent (lowerframe) versus the amplitude $F$ with the parameters $\mu=0.05, \lambda=1, \varepsilon=0.02$ and $2 \omega_{1}-\omega_{2} \simeq 1$ 

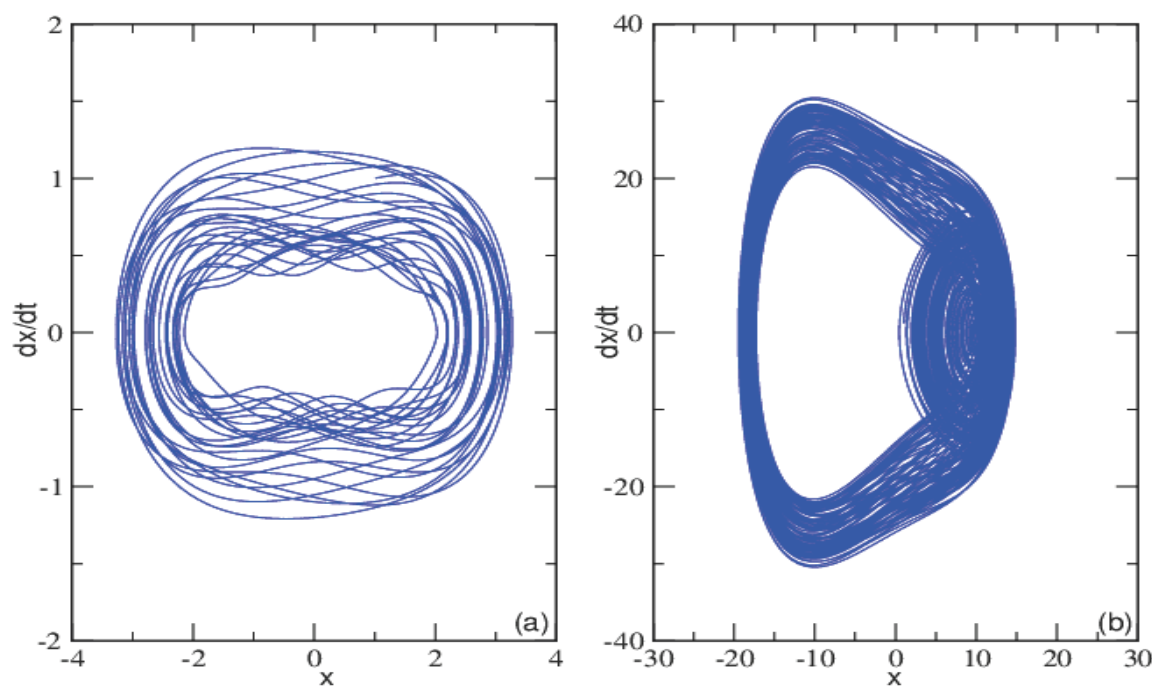

Figure7. Various phase portraits for several different values of $F$ with theparameters of Figure 5
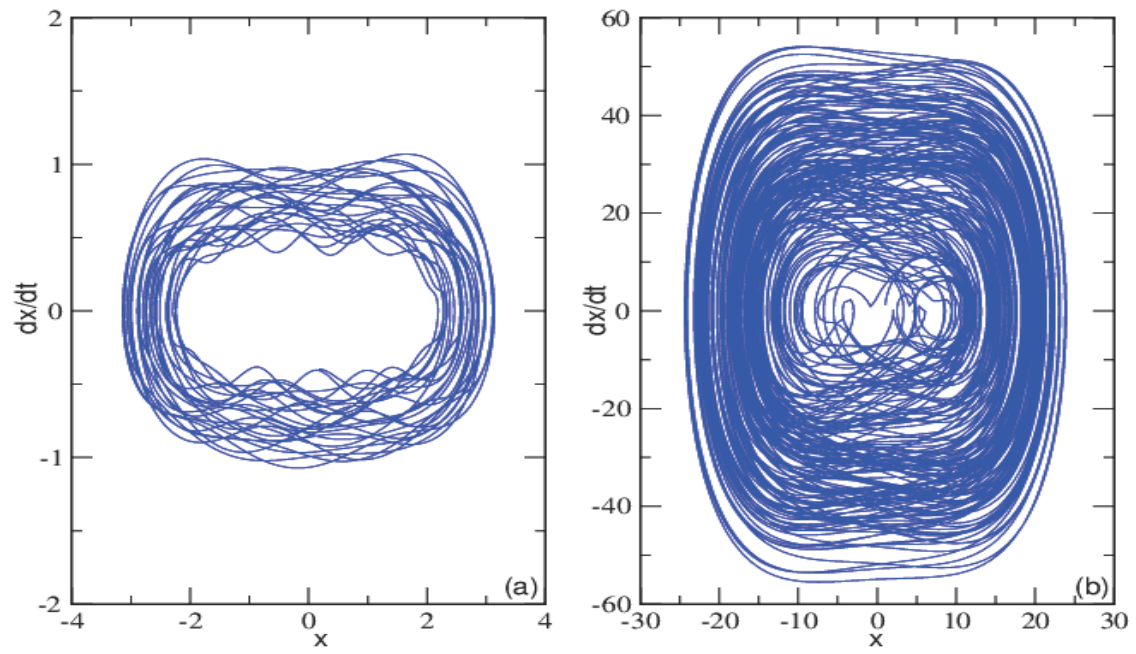

Figure 8. Various phase portraits for several different values of $F$ with the parameters of Figure 6

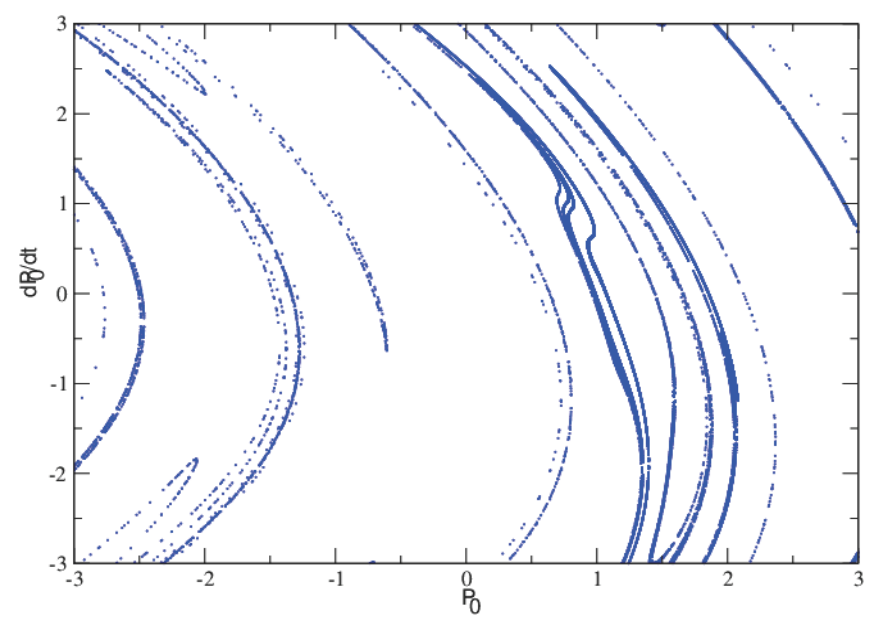

Figure 9. Basin of chaoticity in subharmonic resonant state with $\mu=0.004, \lambda=1, \varepsilon=0.271$ and $\mathrm{F}=20$ 


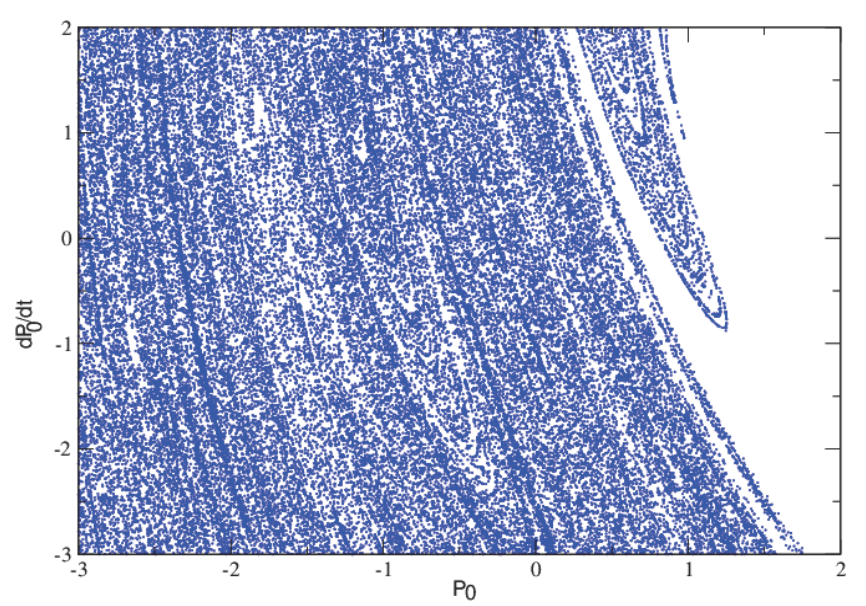

Figure10. Basin of chaoticity in the primary resonant state with $\mu=0.004, \lambda=1, \varepsilon=0.271$ and $\mathrm{F}=16.5$

(a)

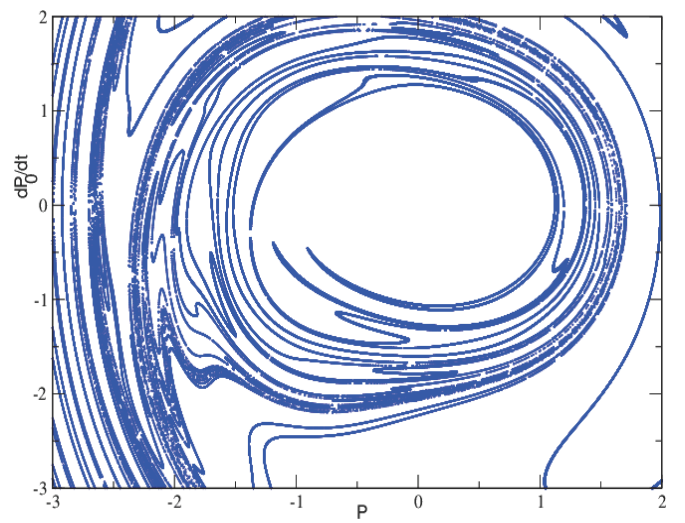

(c)

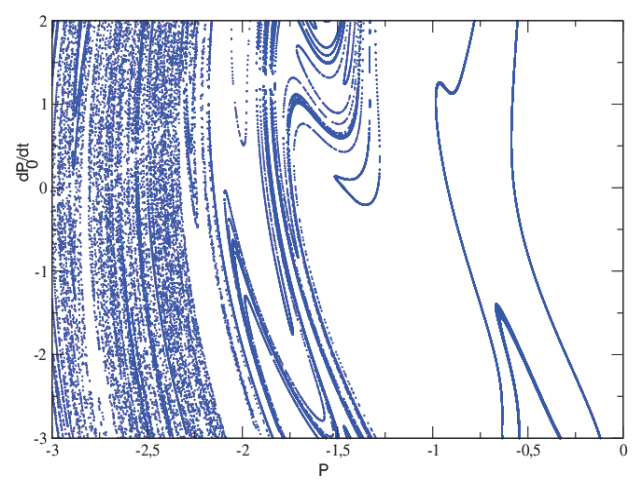

(b)

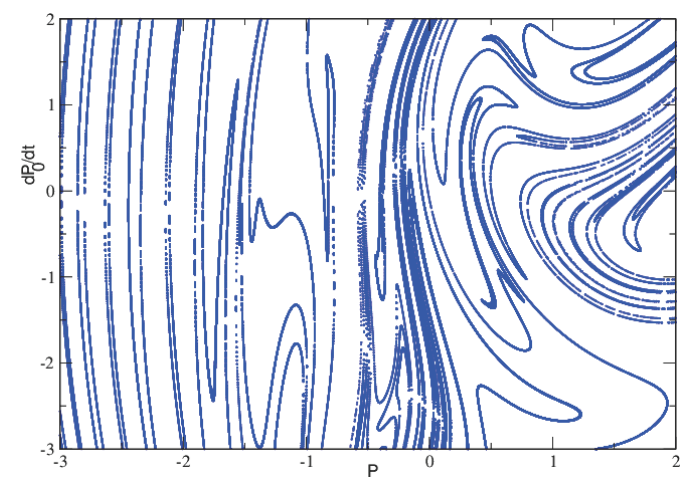

(d)

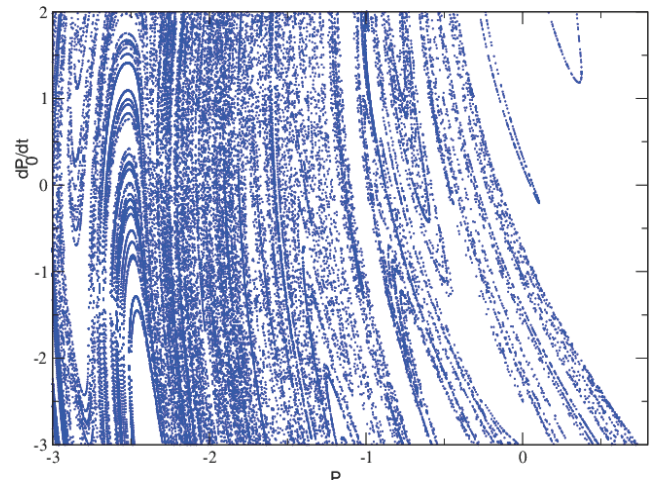

Figure 11. Basin of chaoticity in the secondary resonant state $w_{1}+w_{2} \simeq 2(a) F=0.25,(b) F=3.92,(c) F=$ $10,(d) F=14.1$ with $\mu=0.004, \lambda=1, \varepsilon=0.271$ 
(a)

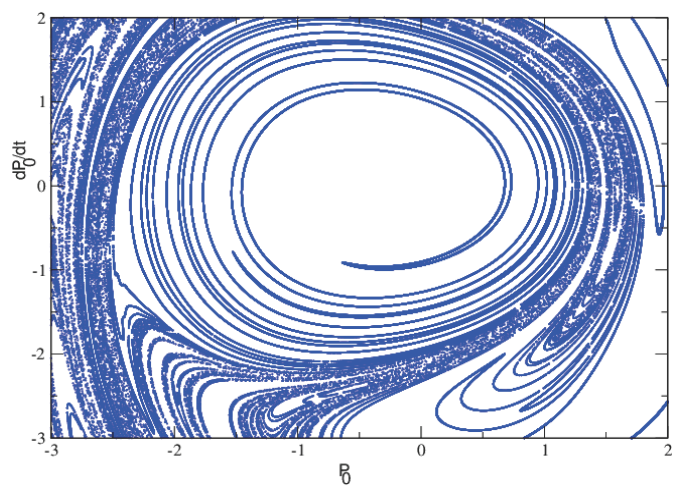

(c)

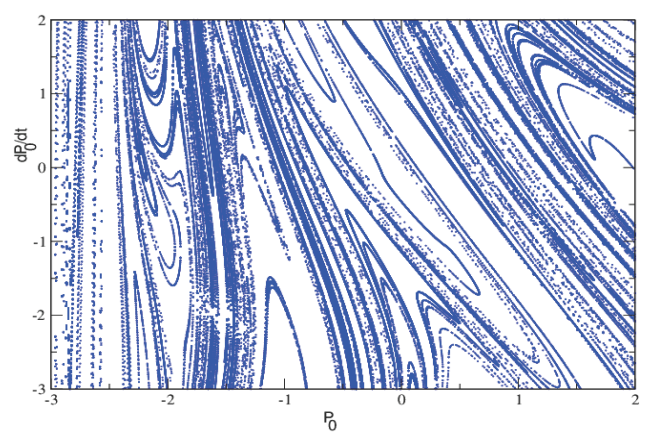

(b)

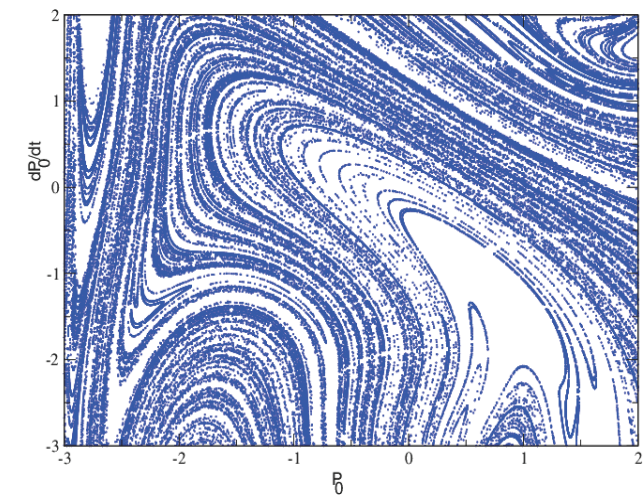

(d)

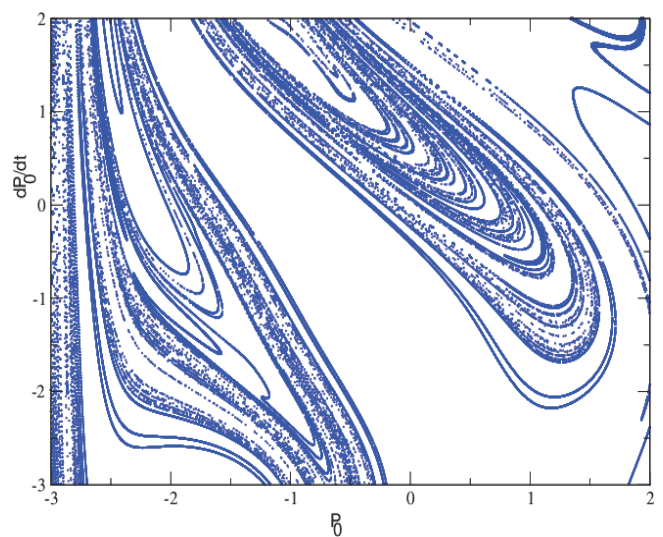

Figure12. Basin of chaoticity in the secondary resonant state $2 w_{1}-w_{2} \simeq 1 ;(a) F=0.25,(b) F=3,(c) F=$ $8.5,(d) F=4.8$ with $\mu=0.004, \lambda=1, \varepsilon=0.271$

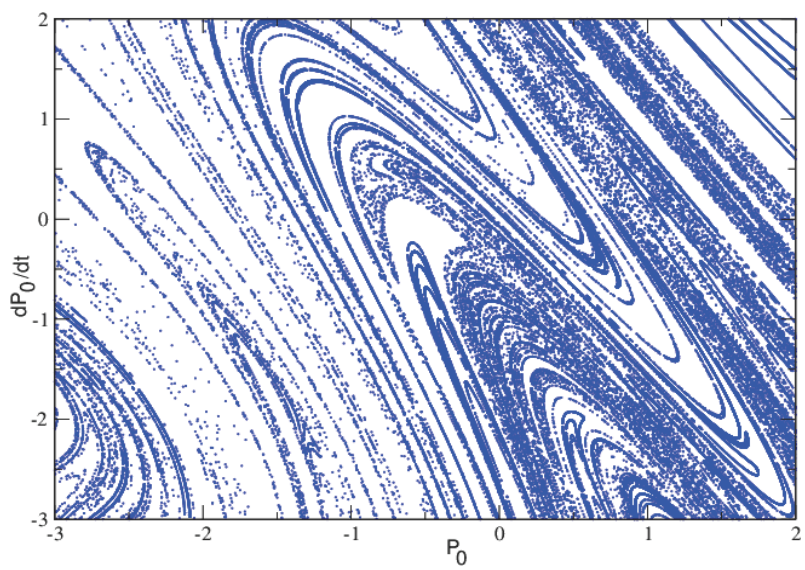

Figure 13. Effect of external frequency on basin of chaoticity in the secondary resonant state $2 w_{1}-w_{2} \simeq 1$ $\left(w_{1}=w_{2}=1\right)$ with $\mathrm{F}=3$ 


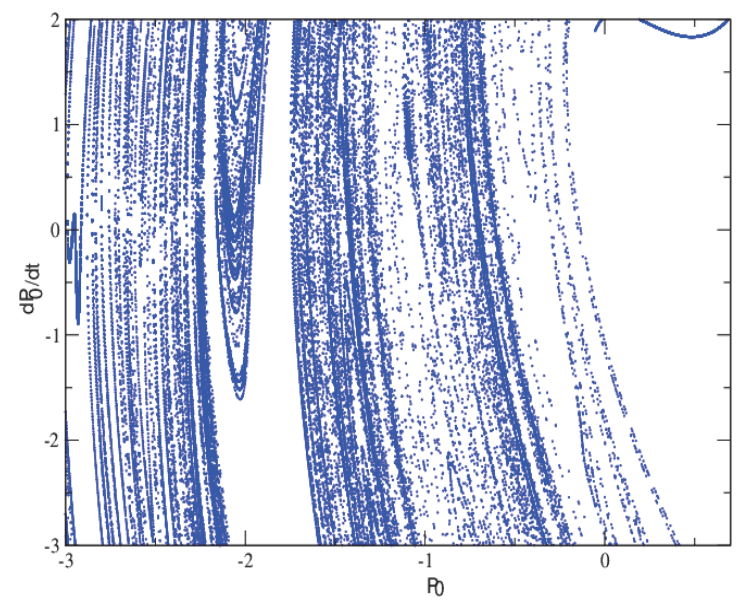

Figure 14. Effect of external frequency on basin of chaoticity in the secondary resonant state $w_{1}+w_{2} \simeq 2$

$$
\left(w_{1}=w_{2}=1\right) \text { with } \mathrm{F}=14.1
$$

\section{Conclusion}

In this paper, we have analyzed the multiresonance and chaotic behaviors of nonlinear dynamics of polarization oscillations when some materials are subjected to the action of an electromagnetic wave modeled by a multifrequency forced Duffing equation. It is obtained by using multiple time scale method apart the cases of superharmonic $\left(3 \omega_{1}=3 \omega_{2}=1+\sigma \varepsilon\right)$ and subharmonic $\left(\omega_{1}=\omega_{2}=3+\sigma \varepsilon\right)$ treated in previous work (Aïnamon, Miwadinou \& Chabi Orou, 2014), primary resonance and three cases for secondary resonances: $\omega_{1}+\omega_{2}=2,2 \omega_{2}-\omega_{1}=1$ and $2 \omega_{1}-\omega_{2}=1$. The jump and hysteresis phenomena are obtained for polarization oscillations. We noticed that for some of these phenomena the system has important singularities which can cause chaotic see catastrophic behavior for the material. This is confirmed by bifurcation diagram and its corresponding Lyapunov exponent. It is found that the polarization oscillations are highly sensitive to the initial conditions through the basin of attraction which are obtained with the external forced amplitude predicted by different bifurcation diagrams. Finally, we noticed that it is possible to use the external frequencies of the electromagnetic wave and parameter $\varepsilon$ to control the material response.

\section{Acknowledgments}

Authors thank EDSM -UAC, IMSP- UAC, ICTP and the state of Benin for financial support. We also thank Laurent Hinvi for his fruitful suggestions and Professor Paul Woafo for his suggestions and collaboration.

\section{References}

Aïnamon, C., Miwadinou, C. H., \& Chabi Orou, J. B. (2014). Resonance study of the polarization of a material subjected to the action of an electromagnetic wave. African Review of Physics (unpublished).

Baltanás, J. P., López, L., Blechman, I. I., Landa, P. S., Zaikin, A., Kurths, J., \& Sanjuán, M. A. F. (2003). Experimental evidence, numerics, and Theory of vibrational resonance in bistable systems. Phys. Rev., E, 67. http://dx.doi.org/10.1103/PhysRevE.67.066119,066119

Boyd, R. (2007). Nonlinear optics (3rd Ed.). Rochester, New York.

Enjieu Kadji, H. G., Chabi Orou, J. B., \& Woafo, P. (2008a). Regular and chaotic behaviors of plasma oscillations modeled by a modified Duffing equation. Phys. Scr., 77(2), 025503. http://dx.doi.org/10.1088/ 0031-8949/77/02/025503

Enjieu Kadji, H. G., Nana Nbendjo, B. R., Chabi Orou, J. B., \& Talla, P. K. (2008b). Nonlinear dynamics of plasma oscillations modeled by an anharmonic oscillator. Phys. Plasmas, 15, 032308. http://dx.doi.org/10.1063/1.2841032

Gammaitoni, L., Hänggi, P., Jung, P., \& Marchesoni, F. (1998). Stochastic resonance, Reviews of Modern Physics, 70(1), 223. http://dx.doi.org/S0034-6861(98)00101-9

Gang, H., Ditzinger, T., Ning, C. Z., \& Haken, H. (1993). Stochastic resonance without external periodic force. Phys. Rev. Lett., 71, 807. http://dx.doi.org/10.1103/PhysRevLett.71.807 
Gouttenoire, V., Barois, T., Perisanu, S., Leclercq, J. L., Purcell, S. T., Vincent, P., \& Ayari, A. (2010). Digital and fm demodulation of a doubly clamped single-walled carbon-nanotube oscillator : Towards a nanotube cell phone. Small, 6(9), 1060-1065. http://dx.doi.org/10.1002/smll.200901984

Nayfeh, A. H. (1981). Introduction to Perturbation Technique. New York: J.Wiley.

Steven, H., \& Strogatz. (1994). Nonlinear Dynamics and Chaos.

Thomas, O., Nezamabadi, S., Lazarus, A., \& Deü, J. F. (2013). Réduction de modèles éléments finis pour les vibrations non linéaires. Application à des nano-structures piézoélectriques. CSMA 2013 11e Colloque National en Calcul des Structures 13-17.

Wiggins, S. (1990). Introduction to Applied Nonlinear Dynamical Systems and Chaos. Texts in Applied Mathematics2.

Yang, J. H., \& Liu, X. B. (2010). Controlling vibrational resonance in a delayed multistable system driven by an amplitude-modulated signal. Phys. Scr., 82, 025006. http://dx.doi.org/10.1088/0031-8949/82/02/025006

Yang, J. H., Sanjuán, M. A. F., Wang, C. J., \& Zhu, H. (2013). Vibrational Resonance in a Duffing System with a Generalized Delayed Feedback. Journal of Applied Nonlinear Dynamics, 2(4), 397-408. http://dx.doi.org/10.5890/JAND.2013.11.006

Yao, C., \& Zhan, M. (2010). Signal transmission by vibrational resonance in one-way coupled bistable systems. Phys. Rev. E, 81, 061129. http://dx.doi.org/10.1103/PhysRevE.81.061129

Yao, C., Liu, Y., \& Zhan, M. (2011). Frequency-resonance-enhanced vibrational resonance in bistable systems, Phys. Rev., E83, 061122. http://dx.doi.org/10.1103/PhysRevE.83.061122

\section{Copyrights}

Copyright for this article is retained by the author(s), with first publication rights granted to the journal.

This is an open-access article distributed under the terms and conditions of the Creative Commons Attribution license (http://creativecommons.org/licenses/by/3.0/). 